\title{
A comparison of two methods of sterile urethral catheterisation in spinal cord injured adults
}

\author{
WG Pickard and DJ Grundy \\ The Duke of Cornwall Spinal Treatment Centre, Salisbury District Hospital, Salisbury, Wilts. SP2 8BJ, UK
}

\begin{abstract}
The purpose of this study was to compare the incidence of urinary infection following sterile catheterisation after either (1) a handwash of $30 \mathrm{~s}$ and double gloving, (the shorter sterile technique) or (2) after a 3 minute wash from fingertips to elbows and the wearing of a sterile gown and one pair of gloves, (the longer sterile technique). Forty-six patients were randomly assigned to the two groups. No significant difference in urinary infection rates was found and as a result the more complicated technique has been discontinued.
\end{abstract}

Keywords: sterile urethral catheterisation; handwashing; spinal cord injury; urinary infection.

\section{Introduction}

It has been the policy of The Duke of Cornwall Spinal Treatment Centre since its inception in 1984 to perform a sterile catheterisation procedure, consisting of a three minute hand-wash from fingertips to elbows and the wearing of a sterile gown. This has proved to be both demanding on the number of nurses required (two per catheterisation), and time consuming due to the procedure itself.

The technique has on the whole been unpopular with nursing staff simply because it was not known whether it reduced the incidence of urinary tract infections. However it would not have been appropriate to abandon the procedure for a simpler and yet still sterile technique if it were to increase urinary infection rates. It was also evident that of the other eleven Spinal Injury Units in Britain, only one other used our procedure at the time of this study. It was not clear which, if any of the two procedures was based on research.

The shorter sterile technique consisted of a brief handwash of not less than $30 \mathrm{~s}$, excluding the forearms, and double gloving. Larson ${ }^{1}$ suggested that $13 \mathrm{~s}$ is adequate, whereas Bowell ${ }^{1,2}$ recommended $30 \mathrm{~s}$. It was not clear from the different studies what was the ideal time to spend on handwashing. Larson ${ }^{1}$ concentrated on its frequency rather than duration, and showed lower bacterial counts on hands when the subjects washed their hands more than eight times per day. Unfortunately the study did not include comparisons of bacterial counts after a longer technique.

The longer sterile technique consisted of a $3 \mathrm{~min}$ wash from fingertips to elbows ${ }^{3}$. Authors fail to agree on the time required - McFarlane ${ }^{4}$ perceived it as

Correspondence: WG Pickard RGN
3 min, whereas an Advanced Operating Room Nurse editorial $^{5}$ acknowledged that the most effective duration for scrubbing is debatable, but is thought to be between five and ten minutes. No researcher discussed the 'hand-scrub' in relation to a clinical setting other than Operating Theatres. Is it necessary to include the forearms of the 'scrub' technique in a clinical setting? Maley ${ }^{6}$ stated:- 'not only are the hands the culprit in spreading pathogens ... forearms also have been found to carry the same pathogens and have been directly linked with nosocomial transfer'. None of these studies were applied to SCI patients.

A literature review showed that although several studies had been undertaken on various hand-washing techniques and types of protective clothing ${ }^{7,8,9}$ no study had been applied to spinal cord injured (SCI) adults; and none compared catheterisation procedures in relation to urinary tract infections.

The hypothesis suggested that a longer sterile catheterisation technique was more likely to decrease the incidence of urinary tract infections in SCI patients than a shorter sterile technique. This seemed a reasonable assumption, after witnessing the elaborate longer technique compared to the shorter procedure.

Allowances had to be made for the possibility that the relationship predicted in the hypothesis did not exist, and to do this a null hypothesis was formulated, ie, there is no relationship between catheterisation techniques and the incidence of urinary tract infections in SCI adults.

It was evident that the most appropriate way to test the hypothesis would be to conduct an experimental study, comparing the incidence of urinary tract infections in controlled and experimental groups, using the two catheterisation procedures. It was hoped that the catheterisation technique that mini- 
mised infection would be revealed, nursing practice being maintained or changed accordingly. Depending on the results this was to be determined by acceptance or rejection of the null hypothesis.

\section{The susceptibility of spinal cord injured patients to urinary tract infection}

SCI patients, whether using an indwelling or intermittent catheter, often have long-standing urinary infections. ${ }^{4}$ Sanderson and Weissler ${ }^{10}$ stated that:'spinal cord injured patients suffer a high incidence of bacteriuria and urinary infection'. Guttmann and Frankel $^{11}$ found 'considerable discrepancy of opinion amongst specialists in this field regarding the best method of preventing infection of the urinary tract in the immediate and early stages following spinal cord injury'. This still applies today and is reflected by the different catheterisation techniques used in Spinal Injury Units throughout Britain. The following practices need to be considered:

\section{(1) Handwashing}

Handwashing is recognised as the single most important procedure in preventing cross-infection in hospital. ${ }^{1,4,6}$ However, is handwashing a priority amongst staff? Larson ${ }^{1}$ found that although nurses wholeheartedly supported its importance in theory, very few actually practised it rigorously.

What of the few who do practice handwashing? Research studies suggest that, though well-meaning, nurses do not do justice to handwashing. Taylor ${ }^{1,2}$ showed some of the inadequacies of handwashing: she blind-folded nurses and asked them to wash their hands using a liquid. The liquid was in fact a dye and showed that few, if any, got dye all over their hands. Whilst studies emphasized the need for thorough handwashing, it was also necessary to review the duration of the handwash.

\section{(2) 'Sterile' versus 'clean' technique}

Guttmann and Frankel $^{11}$ firmly believed in the absolute sterility of the catheterisation procedure. Charbonneau-Smith demonstrated a reduction of $44.5 \%$ with a no touch catheterisation technique using the O'Neil catheter. ${ }^{13}$ Of the studies on SCI patients, Donovan ${ }^{14}$ studied the rate of bacteriuria associated with intermittent catheterisation, whilst Anderson ${ }^{15}$ reported the effect of antibiotic prophylaxis in reducing bacteriuria, using sterile catheterisation. King ${ }^{16}$ compared clean and sterile intermittent catheterisation methods in the absence of prophylactic antibiotic medication. Maynard ${ }^{17}$ repeated Anderson's study using clean, rather than sterile catheterisation. Moore $^{3}$ reviewed these studies and found that the majority of the authors supported clean, rather than sterile catheterisation, and there did not appear to be a lower incidence of bacteriuria when using the sterile technique. She concluded:- "care of the patient with a neurogenic bladder has no absolute answers'.

\section{(3) Protective clothing}

A major factor which may contribute to the neglect of handwashing is the use of sterile gloves. Larson ${ }^{1}$ believed that 'handwashing is often omitted when gloves are worn', and she also stressed the warm, moist environment beneath gloves which aids the rapid multiplication of organisms. Korniewicz ${ }^{16}$ not only found that bacteria can in fact leak through gloves, but that the leakage rate rose to more than fifty per cent as gloves are stressed during use. Barnett ${ }^{7}$ emphasised that even when gloves are worn for a procedure, handwashing should always be viewed as a very important part of that procedure.

Studies published on the different types of protective clothing and their role in cross-infection were aimed at hospital acquired infections and did not concentrate solely on urinary tract infections or SCI patients. Gill ${ }^{9}$ compared different types of protective clothing, removing a patch from each garment, placing them on agar plates and recording bacterial growth at varying intervals. Cotton gowns had bacterial growth almost immediately whereas plastic aprons did not grow any bacteria whatsoever. Their study clearly stated that plastic aprons are for single use. It was not clear whether this rule applied to the cotton gowns before being re-sterilised. Curran ${ }^{8}$ agreed that the use of plastic aprons had been shown to reduce the contamination of nurses' uniforms and, therefore, the patient and his environment. However, she emphasised that this factor alone could not significantly reduce the risk of cross-infection. She concluded that 'effective and frequent handwashing must also be practised'.

Despite the voluminous literature on handwashing, sterile and clean catheterisation techniques, and protective clothing, no studies have been specifically applied to SCI adults using a control and an experimental group to show the incidence of urinary tract infections in relation to two different handwashing techniques.

\section{Methodology}

This study took the form of an experimental approach, as the most appropriate way of comparing handwashing techniques and to test the hypothesis. All the patients treated at The Duke of Cornwall Spinal Treatment Centre over a four month period were studied. The trial was explained to each individual patient and if they were willing to participate verbal consent was obtained by a member of the medical staff. A written record of the patient's consent was entered onto his 'Catheter Trial:Data Collection' form by the doctor concerned. This was adhered to for all patients, even those whose bladder management did not at the time of consent include catheterisation. These patients were included, in case a situation occurred when 
catheterisation became necessary; and if so, the type of procedure would already have been determined and recorded. The intention was to disregard the data on all those patients who did not have catheterisation during the study. Patients who declined to take part in the trial were catheterised according to the Spinal Centre's policy (i.e. the longer procedure, and were not included in the study).

It was important to ensure that all patients involved would have an equal opportunity to have both catheterisation techniques. Therefore, the selection method chosen was that of a 'simple random selection'. Two hundred envelopes, each one containing four identical self-adhesive labels and stating the catheterisation technique, were used. One hundred of these envelopes represented the control group; that is, the longer sterile procedure, and one hundred the experimental group (the shorter sterile procedure). All the nursing staff attended training sessions on how to perform the procedures.

The longer sterile procedure consisted of a three minute wash from fingertips to elbows. Hands and arms were then dried using a separate sterile towel for each arm. The nurse put on a sterile gown and gloves before placing sterile cotton drapes and cleaning the site to be catheterised. The shorter sterile procedure consisted of a simple handwash of not less than $30 \mathrm{~s}$, drying the hands with non-sterile paper towels and wearing a non-sterile plastic apron. Sterile gloves were worn, but instead of several sterile drapes, one sterile disposable lithotomy sheet was used.

Both procedures involved a non-touch catheter technique. Hand cleansing agents used were the same for each technique ie, chlorhexidine gluconate or povidone-iodine solutions. The Centre's policy allows each patient to be individually assessed for the type of cleansing agent, lubricant and catheter to be used. This practice continued during the study. The catheterisation packs for each technique were of equal cost.

Catheter specimens of urine were collected from all patients once a month. In addition specimens were collected on admission to the Centre, at each change of indwelling catheter, and when the patient's symptoms suggested a urinary tract infection.

\section{Results}

At the end of the 4 month trial, the number of patients totalled 94 . Of these, 48 patients' data was disregarded for the following reasons: (1) 35 patients' bladder management had not included catheterisation eg, condom drainage, due to an oversight of the staff, (2) 10 patients were not entered into the trial on their admission to the Spinal Centre: (3) 1 patient declined to take part in the trial: (4) 1 patient's catheterisation technique was changed from one procedure to the other, because the staff could not find the correct sterile pack and (5) 1 patient was admitted from the community with symptoms of urinary tract infection and gross pyuria from a catheter which had been in situ for 6 months.

Therefore, the data used and analysed is that of the remaining 46 patients, with 25 in the control (longer sterile procedure) and 21 in the experimental (shorter sterile procedure) group. Records were kept of the nurse performing each catheterisation, with the intention that the data might highlight human error. However, a pattern could not be found because the same nurse rarely carried out several catheterisations, either to the same patient or to several patients.

Due to the different lengths of stay for each patient, a varying number of specimens were collected per patient. A urinary tract infection was qualified by either of the following two categories:- (1) specimens showing growth and leucocytes of $50 \times 10^{6} /$ litre and above, and (2) patients requiring antibiotics (Table 1). If a patient's microbiology result showed both, it was counted once under 'antibiotic treatment'. Although the categories above were necessary to qualify as a urinary tract infection, additional data was analysed those whose urine specimens showed 'growth and less than 50 leucocytes'; and those showing no growth. As the majority of SCI patients have some degree of bacteriuria, ${ }^{5}$ those with 'growth and less than 50 leucocytes' were not included as a urinary tract infection. Consequently the Spinal Centre only recognizes the two categories already mentioned as denoting a urinary tract infection.

The Chi-squared test was chosen to test the hypothesis because the data fitted its criteria, that is, each group consisted of at least 20 patients; the need to compare the two groups' results for differences; and the data being of the nominal type. No significant difference in urinary infection rates was found in the two groups.

\section{Discussion}

Catheterisation have always been performed as a 'sterile' procedure at The Duke of Cornwall Spinal

Table 1 Contingency Table showing observed frequencies of urinary tract infection rates in relation to the control and experimental groups

\begin{tabular}{lccc}
\hline Group & $\begin{array}{c}\text { Growth of } 50 \times 10^{6} \\
\text { leucocytes/litre }\end{array}$ & $\begin{array}{c}\text { Antibiotic } \\
\text { therapy }\end{array}$ & Total \\
\hline Control & $46(48)$ & $10(8)$ & 56 \\
Experimental & $35(33)$ & $4(6)$ & 39 \\
Total & 81 & 14 & 95 \\
\hline
\end{tabular}

N.B. The expected frequencies are in brackets. The Chi-squared test statistic was calculated using the formula:

$$
\mathrm{x}^{2}=\frac{(\mathrm{o}-\mathrm{E})^{2}}{\mathrm{E}}
$$

where $\mathrm{o}=$ the observed frequency and $\mathrm{E}=$ the expected frequency. The Chi-squared statistic $=1.3$. Since the calculated $x^{2}$ did not fall in the rejection region, the null hypothesis was supported at the 0.05 level of significance, and the hypothesis is rejected 
Treatment Centre, Salisbury. It is a technique which has always been unpopular with the nursing staff because of the uncertainty of its ability to minimise urinary tract infections. The procedure was used because SCI patients are particularly susceptible to bacteriuria, ${ }^{6}$ and urinary tract infections are a major cause of morbidity. ${ }^{18}$

The literature review did not reveal any studies in SCI patients comparing catheterisation techniques in relation to urinary tract infections. However, it did reveal numerous papers on 'handwashing', including 'scrubbing', and protective clothing. ${ }^{1,2,4}$ As Guttmann and Frankel found 30 years ago, there are still differences of opinion regarding the best method of preventing urinary tract infection following SCI, which is reflected by the different catheterisation techniques used in Spinal Injury Units. The question tackled was whether there was an absolute answer to be found; Moore $^{3}$ thought not. The hypothesis which was formulated questioned whether a longer sterile catheterisation technique was more likely to decrease the incidence of urinary tract infections in SCI patients than a shorter sterile technique.

\section{Conclusion}

No relationship between the two catheterisation techniques and the incidence of urinary tract infections was found. Therefore, it cannot be said that a longer sterile rather than a shorter sterile catheterisation technique decreases the incidence of urinary tract infections in SCI patients, and it would seem logical to discontinue the more complicated technique; indeed as a result of this study, the shorter procedure has become standard practice in this Centre.

However, these results should not be viewed in isolation. The long-term implications of catheterisation techniques ie. the level of renal function several years after catheterisation are not known. Only with this knowledge will nursing practice be fully based on research rather than tradition, allowing clinicians to make the choice between different catheterisation techniques. $^{3}$

\section{References}

1 Larson E. Handwashing: its essential - even when you wear gloves. American Journal of Nursing 1989; 89: 934-939.

2 Bowell B. Hands up for cleanliness. Nursing Standard 1991; 8 Jan. $6(15 / 16)$ pp. $24-5$.

3 Moore $\mathrm{K} \mathrm{N}$. Intermittent catheterisation: sterile or clean? Rehabilitation Nursing 1991; 16(1): 15-18,33.

$4 \mathrm{McFarlane} \mathrm{A}$. Why do nurses forget to remember handwashing? Professional Nurse 1990; 5: $250-252$.

5 American Operating Room Journal (editorial). Recommended practices: surgical hand scrubs 1990; 52: $830-836$.

6 Maley M P. External handwashing to the forearms. American Journal of Nursing 1989; 11: 1437.

7 Barnett J. Preventive Procedures. Nursing Times 1991; 6 Mar. 87: $66-8$.

8 Curran E. Protecting with plastic aprons. Nursing Times 1991; Sep. 87 (38): $64-8$.

9 Gill J, Slater J. Building barriers against infection. (Use of protective clothing). Journal of Infection Control Nursing Supplement, Nursing Times 1991; 11 Dec. 87 (50): 53 -4.

10 Sanderson P J, Weissler S. The relation of colonization of the perineum to bacteriuria and environmental contamination in spinally injured patients. Journal of Hospital Infection 1990; 15: $229-234$

11 Guttmann L, Frankel H L. The value of intermittent catheterisation in the early management of traumatic paraplegia and tetraplegia. Paraplegia 1966; 4: 63-82.

12 Taylor L J. An evaluation of handwashing techniques 1 and 2 . Nursing Times 1978; 74: 54 and 74: 108.

13 Charbonneau-Smith R. No-touch catheterisation and infection rates in a select spinal cord injured population. Rehabilitation Nursing 1993; 18: 5.

14 Donovan W H, Stolor W C, Clowers D E, Clowers M R. Bacteriuria during intermittent catheterisation following spinal cord injury. Archives of Physical Medicine and Rehabilitation 1978; 59: 351 - 357 .

15 Anderson,R.U. Non-sterile intermittent catheterisation with antibiotic prophylaxis in the acute spinal cord injured male patient. Journal of Urology 1980; 124: 392-393.

16 King R B, Carlson C, Mervine J, Wu Y, Yarkony G. Clean and sterile catheterisation methods in hospitalized patients with spinal cord injury. Archives of Physical Medicine and Rehabilitation 1992; 73: $798-802$.

17 Maynard F M, Diokno A. Urinary infection and complications during clean intermittent catheterisation following spinal cord injury. Journal of Urology 1984; 132: 943 - 946.

18 Korniewicz D M, Laughton D, Butz A, Larson E. Integrity of vinyl and latex procedure gloves. Nursing Research 1989; 38: $144-146$. 